\title{
Adipose-Derived Fatty Acid-Binding Proteins Plasma Concentrations Are Increased in Breast Cancer Patients
}

\author{
Sandra Guaita-Esteruelas, ${ }^{\text {a,b,c }}$ Paula SaAvedra-García, ${ }^{\text {b,d }}$ Alba Bosquet, ${ }^{\text {a,b }}$ Joan Borràs, ${ }^{\text {c Josefa Girona, }}$ \\ Kepa Amiliano, ${ }^{\mathrm{c}}$ Marta Rodríguez-Balada, ${ }^{\mathrm{c}}$ Mercedes Heras, ${ }^{\mathrm{b}}$ luís Masana, ${ }^{\mathrm{b}}$ Josep Gumà ${ }^{\mathrm{c}}$ \\ ${ }^{a}$ Institut d'Investigació Sanitària Pere Virgili (IISPV), Centre d'R +D+I en Nutrició i Salut, Avda. de la Universitat, Reus, Spain; ${ }^{b}$ Research Unit \\ on Lipids and Atherosclerosis, Universitat Rovira i Virgili, Sant Llorenç, Reus, Spain; 'Institut d'Oncologia de la Catalunya Sud (IOCS), Hospital

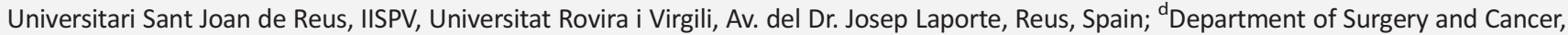 \\ Imperial Centre for Translational and Experimental Medicine (ICTEM), Imperial College London, London, United Kingdom \\ Disclosures of potential conflicts of interest may be found at the end of this article.
}

Key Words. Lipid chaperones • Lipid metabolism • Breast cancer • Adipokines

\section{ABSTRACT}

Background. Adipose tissue is an endocrine organ that could play a role in tumor progression via its secreted adipokines. The role of adipose-derived fatty acid-binding protein (FABP) 4 and FABP5 in breast cancer is presently under study, but their circulating levels in this pathology are poorly known. We analyzed the blood concentrations of FABP4 and FABP5 in breast cancer patients to determine whether there is an association between them and breast cancer.

Materials and Methods. We studied 294 women in the oncology department with a family history of breast cancer; 198 of the women had breast cancer, and 96 were healthy controls. The levels of FABP4, FABP5, lipid profile, standard biochemical parameter, and high-sensitivity C-reactive protein (hSCRP) were determined. We analyzed the association of FABP4 and FABP5 with breast cancer, while adjusting for demographic, anthropometric, and biochemical parameters.

Results. Breast cancer patients had a $24.8 \%(p<.0001)$ and $11.4 \%(p<.05)$ higher blood concentration of FABP4 and FABP5, respectively. Fatty acid-binding protein 4 was positively associated with age, body mass index (BMI), FABP5, very-low-density lipoprotein cholesterol (VLDLC), non-highdensity lipoprote in cholesterol (non-HDLc), Apolipoprotein B 100 (ApoB100), triglycerides, glycerol, glucose, and hsCRP $(p<.05)$, and was negatively associated with HDLc $(p<.005)$ in breast cancer patients. Fatty acid-binding protein 5 was positively associated with BMI, FABP4, VLDLc, triglycerides, glycerol, and hsCRP $(p<.05)$, and was negatively associated with HDLc and Apolipoprotein Al (ApoAI) $(p<.05)$ in breast cancer patients. Using a logistic regression analysis and adjusting for age, BMI, hsCRP, non-HDLc, and triglycerides, FABP4 was independently associated with breast cancer (odds ratio [OR]: 1.091 [95\% Cl: 1.037-1.149]). Moreover, total cholesterol, VLDLc, non-HDLc, ApoB100, triglycerides, and hsCRP were significantly increased in breast cancer patients $(p<.005)$. In contrast, the non-esterified fatty acids concentrations were significantly decreased in breast cancer patients $(p<.05)$.

Conclusion. Circulating FABP4 and FABP5 levels were increased in breast cancer patients compared with controls. The positive association of FABP4 with breast cancer was maintained after adjusting for important covariates, while the association with FABP5 was lost. Our data reinforce the role of adipose tissue and their adipokines in breast cancer. Despite these data, further studies must be performed to better explain the prognosis or diagnostic value of these blood parameters and their possible role in breast cancer. The Oncologist 2017;22:1309-1315

Implications for Practice: We focus on the effect of adipose tissue on cancer, which is increasingly recognized. The association between adipocyte-derived adipokines and breast cancer opens new diagnosis and therapy perspectives. In this study, we provide original data concerning FABP4 and FABP5 plasma concentrations in breast cancer patients. Compared to control group, breast cancer patients show higher FABP4 and FABP5 blood levels. Our data suggest that, particularly, circulating FABP4 levels could be considered a new independent breast cancer biomarker. Our work translates basic science data to clinic linking the relationship between adipose tissue and lipid metabolism to breast cancer.

Correspondence: Sandra Guaita-Esteruelas, Ph.D., Sant Llorenç, 21 Street, 43201 Reus (Spain). Telephone: 34977759315; e-mail: sandra.guaita@ urv.cat Received December 5, 2017; accepted for publication May 18, 2017; published Online First on July 12, 2017. http://dx.doi.org/10.1634/ theoncologist.2016-0483

This is an open access article under the terms of the Creative Commons Attribution-NonCommercial-NoDerivs License, which permits use and distribution in any medium, provided the original work is properly cited, the use is non-commercial and no modifications or adaptations are made. 


\section{INTRODUCTION}

Breast cancer is the most frequent cancer in women in developed countries. Although the mortality rate is decreasing, it remains the most common cause of cancer death in women [1].

Epidemiological studies have demonstrated that patients with diabetes, metabolic syndrome, overweight, and obesity have an increased risk of breast cancer [2-5].

During the last decade, several studies have indicated that the adipose tissue itself is an endocrine organ that could play a role in tumor growth or differentiation by its secreted adipokines [6]. In addition to exerting these local biological effects, adipokines circulate in the plasma and can perform their effects on distant organs [7]. Thus, emerging research has also begun to focus on the role of these circulating adipocyte-secreted factors in breast cancer $[3,5,8]$.

The fatty acid-binding protein (FABP) family is a group of small proteins that act as intracellular fatty acid transporters [9]. Fatty acid-binding proteins are implicated in the uptake and intracellular storage of fatty acids and the regulation of gene expression, cell proliferation, and differentiation [10]. One member of this family, FABP4, also known as aFABP and aP2, is a low-molecular-weight intracellular lipid transport protein, which transports long-chain fatty acids and various other hydrophobic ligands. Fatty acid-binding protein 4 was described to be expressed by adipocytes and macrophages, although it is also expressed in small quantities in other cell types and tumors [10]. Another interesting member of this family is epidermal fatty acid-binding protein 5 (FABP5). Fatty acid-binding protein 5 is expressed in the skin, liver, brain, mammary glands, and, importantly, in adipocytes and macrophages [10], suggesting a coordinated effect with FABP4 [11].

We and other groups have described that circulating FABP4 levels are increased in metabolic pathologies, such as obesity, metabolic syndrome, and type 2 diabetes, and the FABP4 levels could be a useful circulating biomarker for these and other pathologies [12-14]. The data about circulating FABP5 and its clinical implications are more limited. The FABP5 concentration is associated with obesity and metabolic disorders in animal models [15], and some studies have suggested an association of FABP5 with metabolic risk $[11,16]$. Nothing is known about circulating FABP5 and breast cancer.

Regarding the possible function of these FABPs in breast cancer, it has been described that exogenous FABP4 (eFABP4) controls the cell proliferation and migration of human smooth muscle cells from the coronary artery [17]. Exogenous FABP4 activates the cell proliferation of breast cancer cells and induces FABP5 and cluster of differentiation 36 (CD36) expression in MCF7 cells [18]. Moreover, the overexpression of human epidermal growth factor receptor 2 (HER2) increases FABP4 levels [19], and FABP4 expression is high in HER2-positive breast tumors [20]. These results suggest a role for circulating FABP4 in breast cancer. In fact, high circulating FABP4 levels are associated with obesity, breast cancer risk, and adverse tumor characteristics [21].

However, FABP5 plays a key role in breast cancer, functioning in proliferation pathways, retinoic acid-resistant pathways, and the enhancement of epidermal growth factor receptor (EGFR) stability [22-29]. Despite these data, little is known about the circulating levels of FABP4 and FABP5 in breast cancer. Given the various aforementioned functions of FABP4 and FABP5, these proteins could be very good targets for the prevention or treatment of some types of cancer [30].

We studied the association of the plasma levels of FABP4 and FABP5 with the presence of breast cancer and determined whether these levels are linked to breast cancer. We hypothesized that circulating FABP4 and FABP5 may be a breast cancer biomarker.

\section{MaterialS AND METHODS}

\section{Studied Population}

We studied individuals who visited the oncology department of our hospital for breast cancer diagnostics. Breast cancer patients were selected from families undergoing genetic testing at the Genetic Counselling Unit of the South Catalonian Oncology Institute and were non-carriers of their familial BRCA1 or $B R C A 2$ gene mutations. All patients were analyzed in the same group regardless of tumor stage and histological subtype. Control subjects were healthy individuals of the same cohort: noncarriers of BRCA1 and BRCA2 gene mutations. Two hundred ninety-four individuals were included in this study comprising breast cancer patients $(n=198)$ and control subjects $(n=96)$. Anamnesis and physical examination data were recorded. The Hospital Ethical Committee approved the project (reference number: 99-05-20/04-5), and all of the patients provided their written consent to participate in the study and agreed with the publication of the results.

The body mass index (BMI) was calculated as the body weight $(\mathrm{kg})$ divided by the body height squared $\left(\mathrm{m}^{2}\right)$.

\section{Blood Sample Collection and Storage}

A blood sample was obtained after overnight fasting. Plasma aliquots were prepared and stored at $-80^{\circ} \mathrm{C}$ in the BiobankInstitut d'Investigació Sanitària Pere Virgili (IISPV) (recognized in the National Biobank Network Instituto de Salud Carlos III (ISCIII)) of our center until further use. The blood samples were taken before any treatment.

\section{Biochemical Analysis}

The levels of plasma glucose, total cholesterol, high-density lipoprotein cholesterol (HDLc), low-density lipoprotein cholesterol (LDLC), very-low-density lipoprotein cholesterol (VLDLc), triglycerides, ApoB100, ApoAl, high-sensitivity C-reactive protein (hsCRP), and non-esterified fatty acids (NEFA) were analyzed using standard enzymatic and colorimetric techniques (Spinreact, S.A., Girona, Spain, http://www.spinreact.com; Wako Chemicals GmbH, Neuss, Germany, http://www.wako-chemicals.de; Polymedco, Cortlandt, NY, http://www.polymedco. com; coefficient of variation (CV), 4\%) adapted to a Cobas Mira Autoanalyzer (Roche Diagnostics, Sant Cugat del Vallès, Spain, http://www.roche.es). The lipid profile was analyzed according to Spintrol " $\mathrm{H}$ " CAL and GC-MS reference methods. Spintrol " $\mathrm{H}$ " Normal was used as a quality control. Fatty acid-binding protein 4 and FABP5 were evaluated using a commercial ELISA kit (Biovendor, Brno, Czech Republic, http://www.biovendor. com; CV, 5\%). Moreover, we analyzed free glycerol using the glycerol detection kit (Zenbio, Tebu-Bio, Barcelona, Spain, http://www.zen-bio.com). 
Table 1. Characteristics of the study group

\begin{tabular}{|c|c|c|c|}
\hline Study group data & $\begin{array}{l}\text { Control }(n=96), \\
n \text { (range) }\end{array}$ & $\begin{array}{l}\text { Breast cancer } \\
\text { ( } n=198), \\
n \text { (range) }\end{array}$ & $p$ value \\
\hline \multicolumn{4}{|l|}{ Clinical data } \\
\hline Age (years) & $48(38-58)$ & $44(37-50)$ & .062 \\
\hline Number of children & $2(1-2)$ & $2(1-2)$ & .520 \\
\hline Menarche (years) & $12(11-13)$ & $12(12-14)$ & .218 \\
\hline Menopause (yes, \%) & 35.9 & 20.7 & $<.05$ \\
\hline Smoke (yes, \%) & 35.7 & 33.6 & .875 \\
\hline BMI $\left(\mathrm{kg} / \mathrm{m}^{2}\right)$ & $24.0(21.9-28.1)$ & $25.0(22.9-28.6)$ & .078 \\
\hline Hypertension (yes, \%) & 10.1 & 17.0 & .183 \\
\hline Type 2 diabetes (yes, \%) & 20.8 & 20.6 & .329 \\
\hline Obesity (yes, \%) & 12.3 & 18.9 & .270 \\
\hline \multicolumn{4}{|l|}{ Biochemical data } \\
\hline Total cholesterol (mmol/L) & $4.96 \pm 0.90$ & $5.40 \pm 0.99$ & $<.001$ \\
\hline $\mathrm{HDLc}(\mathrm{mmol} / \mathrm{L})$ & $1.65(1.40-1.88)$ & 1.65 (1.39-1.91) & .844 \\
\hline LDLc (mmol/L) & $3.255(2.65-3.81)$ & $3.21(2.56-3.97)$ & .899 \\
\hline VLDLc (mmol/L) & $0.41(0.31-0.67)$ & $0.55(0.40-0.79)$ & $<.001$ \\
\hline Non-HDLc (mmol/L) & $3.26 \pm 0.81$ & $3.71 \pm 0.92$ & $<.0001$ \\
\hline ApoAl (mg/dL) & $140(126-161)$ & 147 (130-165) & .174 \\
\hline ApoB100 (mg/dL) & $93.2 \pm 21.7$ & $101.8 \pm 23.0$ & $<.05$ \\
\hline Triglycerides (mmol/L) & 0.91 (0.69-1.49) & $1.25(0.90-1.77)$ & $<.001$ \\
\hline Glycerol ( $\mu \mathrm{M})$ & $53.6(42.9-73.2)$ & 58.9 (44.6-83.9) & .05 \\
\hline NEFA ( $\mu \mathrm{mol} / \mathrm{L})$ & $478(288-646)$ & $351(231-592)$ & $<.05$ \\
\hline Glucose (mg/dL) & 90.5 (79.5-101.5) & 94.0 (82.0-105.0) & .148 \\
\hline hsCRP (mg/L) & $0.355(0.10-0.81)$ & $0.56(0.16-1.57)$ & $<.001$ \\
\hline
\end{tabular}

Data are expressed as median (IQR) for non-normally distributed data, the mean \pm SD for normally distributed data, or percentages for categorical variables. The statistical tests used were Student's $t$ test (for data that were normally distributed), Mann-Whitney $U$ test (for data that were not normally distributed), or chi-square tests (for data gathered as categorical variables).

Abbreviations: ApoAl, apolipoprotein Al; ApoB100, apolipoprotein B100; BMI, body mass index; HDLc, high-density lipoprotein cholesterol; hsCRP, high-sensitivity C-reactive protein; IQR, interquartile range; LDLc, low-density lipoprotein cholesterol; NEFA, non-esterified fatty acids; Non-HDLc, non-high-density lipoprotein cholesterol; SD, standard deviation; VLDLc, very low-density lipoprotein cholesterol.

\section{Statistical Analysis}

The results were expressed as the mean \pm standard deviation (SD) for normally distributed data, the median (interquartile range) for data that were not normally distributed, and frequencies for categorical data. The differences between groups were assessed using Student's $t$ test, the Mann-Whitney $U$ test, or chi-square tests. Correlations were performed using the Spearman's test. Multiple linear regression analysis using the enter method was used to test the association of the FABP4 and FABP5 concentrations with breast cancer, age, BMI, hsCRP, non-HDLc, and triglycerides. Binary logistic regression analysis with enter method was used to calculate the odds ratio (OR) of serum FABP4 and FABP5 for their association with the presence of breast cancer.

Statistical analyses were performed using SPSS software (IBM SPSS Statistics, version 20.0, North Castle, New York, http://www.ibm.com). A $p$ value $<.05$ was considered to be statistically significant in all analyses.

\section{RESULTS}

Table 1 shows the clinical and demographic characteristics of our cohort of 198 patients and 96 controls. We found a statistically significant difference in the menopause status of control women (35.9\%) compared with breast cancer patients $(20.7 \%$, $p<.05)$. The median age of our breast cancer population was 44 years (range: $37-50$ years; $p=.062$; non-significant $(n . s)$ ) and the BMI was 25.0 (range: 22.9-28.6; $p=.078$; non-significant (n.s)). Additionally, we found no significant differences in number of children, age of menarche, smoke, hypertension, type 2 diabetes, and obesity.

Regarding lipid parameters, total cholesterol, VLDLc, nonHDLc, ApoB100, and triglycerides were significantly increased in breast cancer patients $(p<.005)$. The glycerol concentration was increased in breast cancer patients compared with that in control women (58.93 [44.64-83.93] $\mu \mathrm{M}$ vs. 53.57 [42.8673.21] $\mu \mathrm{M} ; p=.059 ;$ n.s.), but this increase was not significant. Non-esterified fatty acids concentrations were significantly decreased in breast cancer patients (351 [231-592] $\mu \mathrm{mol} / \mathrm{L}$ compared with 478 [288-646.5] $\mu \mathrm{mol} / \mathrm{L}$ in healthy women; $p<.05)$. Moreover, the hsCRP levels were higher in breast cancer patients than in the control cohort $(0.56[0.16-1.57] \mathrm{mg} / \mathrm{L}$ vs. 0.35 [0.10-0.81] mg/L, respectively; $p<.001)$. We found no differences in glucose concentration.

Interestingly, breast cancer patients had a mean increase of 24.8\% $(p<.0001)$ in FABP4 and $11.4 \%(p<.05)$ in FABP5 (Fig. 1) compared with controls. Fatty acid-binding protein 4 


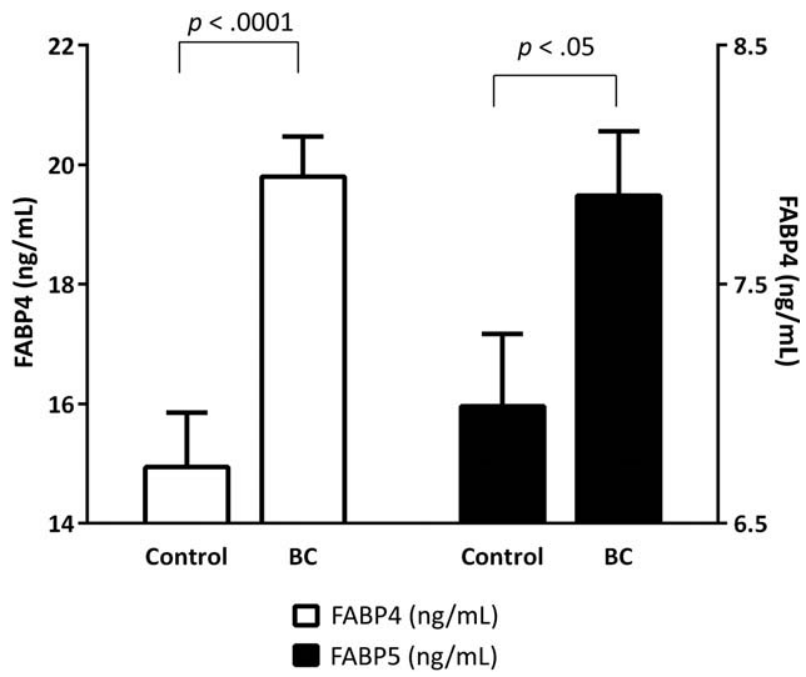

Figure 1. FABP4 and FABP5 plasma concentrations in breast cancer patients and control women. The white bars represent FABP4 levels $(\mathrm{ng} / \mathrm{mL})$, and the black bars represent FABP5 levels $(\mathrm{ng} / \mathrm{mL})$. The bars depict the mean and standard error of the mean. A $p$ value $<.05$ was considered to be statistically significant. Mann-Whitney $U$ test. Abbreviations: BC, breast cancer; FABP, fatty acid-binding protein.

Table 2. Spearman correlation of clinical and biochemical variables with plasma levels of FABP4

\begin{tabular}{|c|c|c|c|c|}
\hline Variable & $\begin{array}{l}\text { Control } \\
(n=96)\end{array}$ & $p$ value & $\begin{array}{l}\text { Breast cancer } \\
\quad(n=198)\end{array}$ & $p$ value \\
\hline Age & 0.402 & $<.0001$ & 0.157 & $<.05$ \\
\hline Menarche & -0.021 & .872 & -0.068 & .407 \\
\hline BMI & 0.452 & $<.0001$ & 0.491 & $<.0001$ \\
\hline FABP5 & 0.389 & $<.0001$ & 0.355 & $<.0001$ \\
\hline Total cholesterol & 0.374 & $<.0001$ & 0.057 & .428 \\
\hline HDLC & 0.019 & .858 & -0.203 & $<.001$ \\
\hline LDLC & 0.335 & $<.001$ & 0.077 & .286 \\
\hline VLDLC & 0.419 & $<.0001$ & 0.314 & $<.0001$ \\
\hline Non-HDLC & 0.376 & $<.0001$ & 0.135 & $<.05$ \\
\hline ApoAl & 0.044 & .681 & -0.097 & .175 \\
\hline АроB100 & 0.398 & $<.0001$ & 0.153 & $<.05$ \\
\hline Triglycerides & 0.419 & $<.0001$ & 0.320 & $<.0001$ \\
\hline Glycerol & 0.270 & $<.05$ & 0.287 & $<.0001$ \\
\hline NEFA & 0.106 & .314 & 0.139 & .053 \\
\hline Glucose & 0.073 & .489 & 0.181 & $<.05$ \\
\hline hsCRP & 0.514 & $<.0001$ & 0.373 & $<.0001$ \\
\hline
\end{tabular}

Abbreviations: ApoAl, apolipoprotein $\mathrm{Al}$; ApoB100, apolipoprotein B100; BMI, body mass index; FABP, fatty acid-binding protein; HDLc, high-density lipoprotein cholesterol; hsCRP, high-sensitivity C-reactive protein; LDLc, low-density lipoprotein cholesterol; NEFA, nonesterified fatty acids; Non-HDLc, non-high-density lipoprotein cholesterol; VLDLc, very low-density lipoprotein cholesterol.

remained significant after adjusting for age, $\mathrm{BMI}$, total cholesterol, triglycerides, NEFA, and hsCRP $(p<.0001)$. When we added menopause status, the difference in FABP4 remain significant $(p<.001)$. By contrast, the FABP5 differences were lost after adjusting for these variables $(p=.323)$. When we stratified by molecular subtype or stage, FABP4 was significantly increased in patients with luminal $A$ and $B$ breast cancer
Table 3. Spearman correlation of clinical and biochemical variables with plasma levels of FABP5

\begin{tabular}{lrccc}
\hline Variable & $\begin{array}{r}\text { Control } \\
(\boldsymbol{n}=\mathbf{9 6})\end{array}$ & $\boldsymbol{p}$ value & $\begin{array}{c}\text { Breast cancer } \\
(\boldsymbol{n}=\mathbf{1 9 8})\end{array}$ & $\boldsymbol{p}$ value \\
\hline Age & 0.217 & $<.05$ & 0.082 & .256 \\
Menarche & 0.083 & .511 & 0.048 & .561 \\
BMI & 0.367 & $<.001$ & 0.251 & $<.001$ \\
FABP4 & 0.389 & .0001 & 0.355 & .0001 \\
Total & 0.191 & .063 & -0.019 & .785 \\
cholesterol & & & & \\
HDLc & -0.083 & .420 & -0.213 & $<.001$ \\
LDLc & 0.209 & $<.05$ & -0.033 & .641 \\
VLDLc & 0.285 & $<.05$ & 0.193 & $<.05$ \\
Non-HDLc & 0.266 & $<.05$ & 0.060 & .397 \\
ApoAl & -0.078 & .455 & -0.170 & $<.05$ \\
ApoB100 & 0.240 & $<.05$ & 0.059 & .407 \\
Triglycerides & 0.285 & $<.001$ & 0.206 & $<.001$ \\
Glycerol & 0.275 & $<.001$ & 0.240 & $<.001$ \\
NEFA & 0.069 & .504 & 0.043 & .546 \\
Glucose & 0.081 & .435 & -0.076 & .289 \\
\hline hsCRP & 0.185 & .071 & 0.276 & .0001 \\
\hline Abbreviation & Ap & & & \\
\hline
\end{tabular}

Abbreviations: ApoAl, apolipoprotein Al; ApoB100, apolipoprotein B100; BMI, body mass index; FABP, fatty acid-binding protein; HDLC, high-density lipoprotein cholesterol; hsCRP, high-sensitivity C-reactive protein; LDLc, low-density lipoprotein cholesterol; NEFA, non-esterified fatty acids; Non-HDLc, non-high-density lipoprotein cholesterol; VLDLc, very low-density lipoprotein cholesterol.

subtype with respect to control group ( $p=.026$ and $p=.005$, respectively). Moreover, a significant increase in FABP4 was observed in stage II and III with respect to control group ( $p=.001$ and $p=.005$, respectively).

Table 2 shows the correlations between the FABP4 plasma concentrations and biochemical data in breast cancer patients and control women. Fatty acid-binding protein 4 was significantly positively associated with age, BMI, FABP5, total cholesterol, LDLc, VLDLc, non-HDLc, ApoB100, triglycerides, glycerol, and hsCRP $(p<.05)$ in control population. When we studied the correlation in the breast cancer group, the significant association between FABP4 and total cholesterol and LDLc was lost. Interestingly, the significant negative correlation of FABP4 and HDLc $(r=-.203 ; p<.005)$ and the significant positive association with glucose $(r=.181 ; p<.05)$ was only observed in breast cancer patients.

Table 3 shows the correlations between FABP5 plasma concentrations and biochemical data in breast cancer and control women. Fatty acid-binding protein 5 was significantly positively associated with age, BMI, FABP4, LDLc, VLDLc, non-HDLc, ApoB100, triglycerides, and glycerol $(p<.05)$ in control population. When we studied the correlation in the breast cancer group, the significant association between FABP5 and age, LDLc, non-HDLc, and ApoB100 was lost. Interestingly, the significant negative correlation of FABP5 and HDLC $(r=-.213$; $p<.001)$, ApoAl $(r=-.170 ; p<.05)$ and the significant positive association with hsCRP $(r=.276 ; p<.0001)$ was only observed in breast cancer patients.

To further explore the relationship between FABP4 and breast cancer, a linear regression model was generated (Table 4 
Table 4. Linear regression analysis of FABP4 and FABP5 with breast cancer

\begin{tabular}{|c|c|c|c|c|c|c|}
\hline \multirow[b]{2}{*}{ Model } & \multicolumn{3}{|c|}{ FABP4 } & \multicolumn{3}{|c|}{ FABP5 } \\
\hline & Beta & $r^{2}$ & $p$ value & Beta & $r^{2}$ & $p$ value \\
\hline Model 1: BC (crude) & 4.86 & .06 & $<.0001$ & 0.88 & .01 & $<.05$ \\
\hline Model 2: $\mathrm{BC}+$ age & 5.89 & .13 & $<.0001$ & 0.89 & .04 & $<.05$ \\
\hline Model 3: $\mathrm{BC}+$ age $+\mathrm{BMI}$ & 4.80 & .28 & $<.0001$ & 0.68 & .06 & .148 \\
\hline Model 4: $\mathrm{BC}+$ age $+\mathrm{BMI}+\mathrm{hsCRP}$ & 4.29 & .32 & $<.0001$ & 0.62 & .06 & .185 \\
\hline $\begin{array}{l}\text { Model 5: BC }+ \text { age }+ \text { BMI }+ \\
\text { hsCRP }+ \text { non-HDLC }\end{array}$ & 4.16 & .32 & $<.0001$ & 0.58 & .08 & .230 \\
\hline $\begin{array}{l}\text { Model 6: BC }+ \text { age }+ \text { BMI }+ \\
\text { hsCRP + non-HDLc }+ \text { TG }\end{array}$ & 3.95 & .33 & $<.0001$ & 0.48 & .08 & .319 \\
\hline
\end{tabular}

Beta unstandardized coefficients for breast cancer.

Abbreviations: BC, breast cancer; BMI, body mass index; FABP, fatty acid-binding protein; hSCRP, high-sensitivity C-reactive protein; Non-HDLc, non-high-density lipoprotein cholesterol; TG, triglycerides.

Table 5. Logistic regression analysis for variables predicting breast cancer

\begin{tabular}{llll}
\hline Variable & OR & $95 \% \mathbf{C l}$ & $\boldsymbol{p}$ value \\
\hline FABP4 & 1.091 & $1.037-1.149$ & .001 \\
Age & 0.941 & $0.913-0.970$ & .000 \\
BMI & 0.987 & $0.909-1.072$ & .76 \\
Non-HDLc & 1.755 & $1.135-2.714$ & .011 \\
TG & 1.416 & $0.802-2.499$ & .23 \\
FABP5 & 1.035 & $0.938-1.141$ & .491 \\
Age & 0.954 & $0.928-0.981$ & .001 \\
BMI & 1.038 & $0.965-1.117$ & .314 \\
Non-HDLc & 1.702 & $1.13-2.565$ & .011 \\
TG & 1.542 & $0.887-2.678$ & .125 \\
\hline
\end{tabular}

Data are presented as OR $(95 \% \mathrm{Cl})$.

Abbreviations: $\mathrm{BC}$, breast cancer; $\mathrm{BMI}$, body mass index; $\mathrm{Cl}$, confidence interval; FABP, fatty acid-binding protein; hsCRP, high-sensitivity C-reactive protein, Non-HDLc, non-high-density lipoprotein cholesterol; OR, odds ratio; TG, triglycerides.

and supplemental online Table 1). The significant direct relation between FABP4 and breast cancer remained robust after additional adjustment for the following covariates (model 6): age, BMI, hsCRP, non-HDLc, and triglycerides $\left(r^{2}=.331, p<.0001\right)$. No significant association was found between FABP5 and breast cancer after the multiple adjustment (Table 4 and supplemental online Table 1).

Table 5 depicts the OR for FABP4 and FABP5 and selected covariates in breast cancer patients compared with those in control women. The FABP4 concentration was associated with a higher risk of breast cancer after adjusting for age, BMI, nonHDLc, and triglycerides (OR: 1.091 [95\% Cl: 1.037-1.149]). Conversely, the FABP5 concentration was not associated with an increased likelihood of having breast cancer (OR: 1.035 [95\% Cl: 0.938-1.141]). Age and non-HDLc also showed a significant association with breast cancer risk.

\section{DiscuSSION}

In this study, we evaluated the FABP4 and FABP5 plasma concentrations in breast cancer patients. Our main conclusions are that FABP4 and FABP5 are higher in breast cancer patients. This association remained significant after adjusting for BMI, total cholesterol, triglycerides, NEFA, hsCRP, age, and menopause, but the association was lost in the case of FABP5. The FABP4 results are in agreement with previous findings from Hancke et al. [21]. They described that FABP4 was associated with breast cancer risk, and breast cancer patients with high serum levels of FABP4 had a worse prognosis in populations of postmenopausal women. They focused their study on postmenopausal and obese women, but we extended this observation in our populations of $80 \%$ premenopausal patients. Moreover, they showed significantly higher serum FABP4 levels in obese than in nonobese breast cancer patients and controls, independent of menopausal status [21]. Our results provide a new viewpoint to previous ones because our FABP4 association with breast cancer was independent of BMI. Despite these data, there are no other reports describing the association of circulating FABP4 and breast cancer or even other cancer types. Furthermore, some recent publications have addressed the possible function of FABP4 in breast cancer [18-20, 31-33], suggesting a putative role for this molecule in this pathology. These results are emerging with an exciting role for circulating FABP4 in breast cancer progression because circulating FABP4 would play a role in either fatty acid transport or activating key survival and proliferation pathways.

Although some publications also described the cellular role of FABP5 in breast cancer, there are no studies on circulating FABP5 and breast cancer. The role of circulating FABP5 in breast cancer could be important considering that FABP5 has some important functions in breast cancer, such as its participation in proliferation pathways, retinoic acid-resistant pathways, and EGFR stability [22-29]. To our knowledge, this is the first paper describing the increased levels of circulating FABP5 in breast cancer patients. Moreover, we described that the association of FABP5 with breast cancer is lost after adjusting for BMI, total cholesterol, triglycerides, NEFA, hsCRP, age, and menopause. This suggests a cooperative mechanism between these risk factors and FABP5.

Moreover, total cholesterol, VLDLc, non-HDLc, ApoB100, and triglycerides are increased in our cohort of breast cancer patients. Moreover, non-HDLc was associated with breast cancer risk in the logistic regression model. These results agree with previous results in other populations [34-36]. However, we found no significant difference in the HDLc or LDLc levels. There are some controversial results concerning HDLc and LDLC 
in breast cancer, and more studies should be performed to define the association between breast cancer and these molecules [20, 33, 37-39].

Because total cholesterol and non-HDLc, but not LDLc or HDLc, are higher in breast cancer patients in our breast cancer population, we hypothesize that there is a possibility of a new role for VLDLc, which is rich in ApoB100 and triglycerides, in this pathology. In fact, breast cancer patients have higher levels of VLDLc than control women in our cohort.

Fatty acid-binding protein 4 and FABP5 were highly correlated with hSCRP in our population. Interestingly, FABP5 is associated with hsCRP only in our breast cancer population. There are some controversial results regarding the levels and the possible role of CRP in breast cancer. In fact, the CRP levels are elevated under specific conditions in breast cancer patients, but the results are not conclusive [40-42]. In our population, we found that the CRP levels are higher in breast cancer patients, implicating a role of inflammation in this pathology. As described above, FABP4 and FABP5 were also elevated in breast cancer patients, again supporting the role of inflammation in our breast cancer population. In fact, it has been described previously that FABP4 and FABP5 are associated with CRP in some metabolic pathologies $[11,43]$, and we describe these new associations in breast cancer.

Actually, the association between FABP4 and FABP5 and lipid or inflammation parameters has been shown in other metabolic disorders, such as type 2 diabetes, obesity, and metabolic syndrome [11, 13, 43], but they have not been described in breast cancer. Moreover, as we showed in the multivariate analyses, FABP4 is associated with breast cancer after adjusting for age, BMI, non-HDLc, triglycerides, and hsCRP. Thus, FABP4 is an independent biomarker for breast cancer despite other metabolic parameters. In fact, in the bivariate correlation, we showed that, in breast cancer patients, FABP4 is associated positively with age, BMI, FABP5, VLDLc, non-HDLc, ApoB100, triglycerides, glycerol, glucose, and hsCRP, and is negatively associated with HDLc in our breast cancer population. Fatty acid-binding protein 5 is associated positively with BMI, FABP4, VLDLc, triglycerides, glycerol, and hsCRP, and is negatively associated with HDLc and ApoAl in our breast cancer population. This association is lost after adjusting for the covariables described above, showing a dependent mechanism for FABP5 in breast cancer. Finally, we described that FABP4 increases the risk of breast cancer, but we could not find significant differences in FABP5. Obviously, metabolic disorders play important roles in cancer progression; in our breast cancer cohort, FABP5 lost its association with breast cancer when we corrected for some metabolic variables. However, to our knowledge, this is the first time that FABP4 has been described as an independent biomarker for breast cancer.
These results suggest that FABP4 and FABP5 might act as a paracrine adipokine and might be two of the molecules key to understanding the mechanisms underlying the obesity-breast cancer progression link and the cross-talk between cancer cells and adipose tissue in non-obese patients.

Our study has some limitations. Our population is a subgroup of patients belonging to families with a genetic history of breast cancer. Although these patients are non-carriers of BRAC1 and BRCA2 mutation, they could have other genetic alterations that we did not consider. Therefore, this population can introduce a bias in the interpretation of the results and the extrapolation for the sporadic breast cancer risk population. Accordingly, our breast cancer population was younger than controls, explaining the negative association observed between age and $\mathrm{BC}$ in the regression model. Moreover, we have not taken into account the different tumor stage and histological subtypes.

\section{CONCLUSION}

The FABP4 and FABP5 concentrations are higher in breast cancer patients. Fatty acid-binding protein 4 association was independent of age, BMI, lipids, and inflammation status, while FABP5 association was lost after adjusting, suggesting that there is a direct impact of high FABP4 concentration on breast cancer. These results strengthen the role of these adipocytesecreted factors on breast cancer. Further research may provide evidence of the effects of these circulating adipokines on breast cancer.

\section{ACKNOWLEDGMENTS}

This work was partially supported by the grant 2013LINE-05 from the Universitat Rovira Virgili, Banco de Santander, and Generalitat de Catalunya. The funders had no role in study design, data collection and analysis, decision to publish, or preparation of the manuscript.

\footnotetext{
Author Contributions

Conception/design: Sandra Guaita-Esteruelas

Provision of study material or patients: Joan Borràs, Kepa Amiliano, Marta Rodríguez-Balada

Collection and/or assembly of data: Sandra Guaita-Esteruelas, Paula SaavedraGarcía, Alba Bosquet, Kepa Amiliano, Marta Rodríguez-Balada, Mercedes Heras

Data analysis and interpretation: Sandra Guaita-Esteruelas, Paula SaavedraGarcía, Alba Bosquet, Josefa Girona, Mercedes Heras, Luís Masana, Josep Gumà

Manuscript writing: Sandra Guaita-Esteruelas, Josefa Girona, Luís Masana, Josep Gumà

Final approval of manuscript: Sandra Guaita-Esteruelas, Joan Borràs, Luís Masana, Josep Gumà
}

Disclosures

The authors indicated no financial relationships.

\section{REFERENCES}

1. Siegel R, Ma J, Zou Z et al. Cancer statistics, 2014. CA Cancer J Clin 2014;64:9-29.

2. Nimptsch K, Pischon T. Body fatness, related biomarkers and cancer risk: An epidemiological perspective. Horm Mol Biol Clin Investig 2015;22:39-51.

3. Schmidt S, Monk JM, Robinson LE et al. The integrative role of leptin, oestrogen and the insulin family in obesity-associated breast cancer:
Potential effects of exercise. Obes Rev 2015;16:473487.

4. Parkin DM, Boyd L. 8. Cancers attributable to overweight and obesity in the UK in 2010. Br J Cancer 2011;105(suppl 2):S34-S37.

5. Donohoe CL, Doyle SL, Reynolds JV. Visceral adiposity, insulin resistance and cancer risk. Diabetol Metab Syndr 2011;3:12.
6. Housa D, Housová J, Vernerová Z et al. Adipocytokines and cancer. Physiol Res 2006;55:233-244.

7. Vona-Davis L, Rose DP. Adipokines as endocrine, paracrine, and autocrine factors in breast cancer risk and progression. Endocr Relat Cancer 2007;14:189206.

8. Booth A, Magnuson A, Fouts J et al. Adipose tissue, obesity and adipokines: Role in cancer 
promotion. Horm Mol Biol Clin Investig 2015;21: 57-74.

9. Smathers RL, Petersen DR. The human fatty acid-binding protein family: Evolutionary divergences and functions. Hum Genomics 2011;5:170-191.

10. Furuhashi M, Hotamisligil GS. Fatty acidbinding proteins: Role in metabolic diseases and potential as drug targets. Nat Rev Drug Discov 2008; 7:489.

11. Ibarretxe D, Girona J, Amigó $\mathrm{N}$ et al. Impact of epidermal fatty acid binding protein on 2D-NMRassessed atherogenic dyslipidemia and related disorders. J Clin Lipidol 2016;10:330-338.

12. Cabré A, Lázaro I, Girona J et al. Plasma fatty acid binding protein 4 is associated with atherogenic dyslipidemia in diabetes. J Lipid Res 2008;49:17461751.

13. Cabré A, Lázaro I, Girona J et al. Fatty acid binding protein 4 is increased in metabolic syndrome and with thiazolidinedione treatment in diabetic patients. Atherosclerosis 2007;195: e150-e158.

14. Xu A, Wang Y, Xu JY et al. Adipocyte fatty acidbinding protein is a plasma biomarker closely associated with obesity and metabolic syndrome. Clin Chem 2006;52:405-413.

15. Maeda K, Uysal KT, Makowski L et al. Role of the fatty acid binding protein mal1 in obesity and insulin resistance. Diabetes 2003;52:300-307.

16. Yeung DC, Xu A, Tso AW et al. Circulating levels of adipocyte and epidermal fatty acid-binding proteins in relation to nephropathy staging and macrovascular complications in type 2 diabetic patients. Diabetes Care 2009;32:132-134.

17. Girona J, Rosales R, Plana N et al. FABP4 induces vascular smooth muscle cell proliferation and migration through a MAPK-dependent pathway. PLoS One 2013;8:e81914.

18. Guaita-Esteruelas $S$, Bosquet $A$, Saavedra $P$ et al. Exogenous FABP4 increases breast cancer cell proliferation and activates the expression of fatty acid transport proteins. Mol Carcinog 2016;56:208217.

19. Ravacci GR, Brentani MM, Tortelli TC et al. Docosahexaenoic acid modulates a HER2-associated lipogenic phenotype, induces apoptosis, and increases trastuzumab action in HER2overexpressing breast carcinoma cells. Biomed Res Int 2015;2015:838652.
20. Kim S, Lee Y, Koo JS. Differential expression of lipid metabolism-related proteins in different breast cancer subtypes. PLoS One 2015;10:e0119473.

21. Hancke K, Grubeck D, Hauser N et al. Adipocyte fatty acid-binding protein as a novel prognostic factor in obese breast cancer patients. Breast Cancer Res Treat 2010;119:367-377.

22. Zhang W, Levi L, Banerjee $P$ et al. Kruppel-like factor 2 suppresses mammary carcinoma growth by regulating retinoic acid signaling. Oncotarget 2015;6 35830-35842.

23. Liu RZ, Garcia E, Glubrecht DD et al. CRABP1 is associated with a poor prognosis in breast cancer: Adding to the complexity of breast cancer cell response to retinoic acid. Mol Cancer 2015;14:129.

24. Liu RZ, Graham K, Glubrecht DD et al. Association of FABP5 expression with poor survival in triplenegative breast cancer: Implication for retinoic acid therapy. Am J Pathol 2011;178:997-1008.

25. Powell CA, Nasser MW, Zhao $\mathrm{H}$ et al. Fatty acid binding protein 5 promotes metastatic potential of triple negative breast cancer cells through enhancing epidermal growth factor receptor stability. Oncotarget 2015;6:6373-6385.

26. Thulasiraman $P$, McAndrews DJ, Mohiudddin IQ. Curcumin restores sensitivity to retinoic acid in triple negative breast cancer cells. BMC Cancer 2014;14:724.

27. Kannan-Thulasiraman $P$, Seachrist DD, Mahabeleshwar GH et al. Fatty acid-binding protein 5 and PPARbeta/delta are critical mediators of epidermal growth factor receptor-induced carcinoma cell growth. J Biol Chem 2010;285:19106-19115.

28. Levi L, Lobo G, Doud MK et al. Genetic ablation of the fatty acid-binding protein FABP5 suppresses HER2-induced mammary tumorigenesis. Cancer Res 2013;73:4770-4780.

29. Schug $\Pi$, Berry DC, Toshkov IA et al. Overcoming retinoic acid-resistance of mammary carcinomas by diverting retinoic acid from PPARbeta/delta to RAR. Proc Natl Acad Sci U S A 2008;105:7546-7551.

30. Nieman KM, Kenny HA, Penicka CV et al. Adipocytes promote ovarian cancer metastasis and provide energy for rapid tumor growth. Nat Med 2011; 17:1498-1503.

31. Vyberg M, Nielsen S, Røge R et al. Immunohistochemical expression of HER2 in breast cancer: Socioeconomic impact of inaccurate tests. BMC Health Serv Res 2015;15:352.
32. Wang W, Yuan P, Yu D et al. A single-nucleotide polymorphism in the $3^{\prime}$-UTR region of the adipocyte fatty acid binding protein 4 gene is associated with prognosis of triple-negative breast cancer. Oncotarget 2014;7:18984-18998.

33. Jung YY, Kim HM, Koo JS. Expression of lipid metabolism-related proteins in metastatic breast cancer. PLoS One 2015;10:e0137204.

34. Kumar V, Singh A, Sidhu DS et al. A comparitive study to evaluate the role of serum lipid levels in aetiology of carcinoma breast. J Clin Diagn Res 2015; 9:PC01-PC03.

35. Paillasse MR, de Medina P, Amouroux G et al. Signaling through cholesterol esterification: A new pathway for the cholecystokinin 2 receptor involved in cell growth and invasion. J Lipid Res 2009;50: 2203-2211.

36. Abdelsalam KE, Hassan IK, Sadig IA. The role of developing breast cancer in alteration of serum lipid profile. J Res Med Sci 2012;17:562-565.

37. Zhang F, Chen Y, Long J et al. Dietary fatty acids affect lipid metabolism and estrogen receptor expression in $\mathrm{N}$-methyl-N-nitrosourea-induced rat mammary cancer model. Clin Lab 2015;61:389-395.

38. Borrelli R, del Sordo G, De Filippo E et al. High serum HDL-cholesterol in pre- and post-menopausal women with breast cancer in southern Italy. Adv Exp Med Biol 1993;348:149-153.

39. Nieva C, Marro M, Santana-Codina N et al. The lipid phenotype of breast cancer cells characterized by raman microspectroscopy: Towards a stratification of malignancy. PLoS One 2012;7:e46456.

40. Allin KH, Bojesen SE, Nordestgaard BG. Inflammatory biomarkers and risk of cancer in 84,000 individuals from the general population. Int J Cancer 2016;139:1493-1500.

41. Agresti R, Meneghini E, Baili P et al. Association of adiposity, dysmetabolisms, and inflammation with aggressive breast cancer subtypes: A crosssectional study. Breast Cancer Res Treat 2016;157: 179-189.

42. Abdollahi A, Ali-Bakhshi A, Farahani Z. Concentration study of high sensitive $\mathrm{C}$ - reactive protein and some serum trace elements in patients with benign and malignant breast tumor. Int J Hematol Oncol Stem Cell Res 2015;9:180-184.

43. Cabré A, Lázaro I, Cofán M et al. FABP4 plasma levels are increased in familial combined hyperlipidemia. J Lipid Res 2010;51:1173-1178.

See http://www.TheOncologist.com for supplemental material available online. 